\title{
A Bivariate Discrete Distribution from Freund Bivariate Exponential Distribution
}

\author{
S. R. Supanekar \\ Department of Statistics, Balasaheb Desai College, Patan, Dist. Satara, India(MS) 415206 \\ *Corresponding Author: sup_sanjay@yahoo.com, Tel.: +91-7083881971
}

Available online at: www.isroset.org

Accepted 12/Aug/2018, Online 30/Aug/2018

\begin{abstract}
A bivariate discrete distribution is introduced by transforming a bivariate continuous random vector. Continuous random vector has been transformed to a discrete random vector by considering integer part of the components of continuous random vector. Freund bivariate exponential distribution is considered and corresponding bivariate discrete distribution is obtained. The methodology used is simple and can be applied to generate variety of bivariate/multivariate discrete distributions.
\end{abstract}

Keywords - Bivariate discrete distribution; Freund bivariate exponential distribution; Transformation of random variables

\section{INTRODUCTION}

In the last few decades research dealing with discrete distributions obtained by discretizing a continuous distributions. Recently, Gomez-Deniz et al. [1] obtained a discrete version of the half-normal distribution and reported its generalization with applications. Bivariate discrete distributions like Poisson, Geometric, and Binomial etc. play a vital role in analyzing real life situations. The different bivariate distributions are available in the literature. The bivariate Poisson distribution available in the literature due to Holgate [2] and Teicher [3] considers non-trivial cases of multivariate Poisson distribution. There are so many ways to construct bivariate discrete distributions. In the literature, numerous truncated versions of bivariate discrete distributions have been studied. Hamdan [4] and Dahiya [5] have considered truncated bivariate Poisson distribution. Some other methods to construct bivariate discrete distributions are convolution and copula. The best references of different copula and an extensive account of bivariate discrete distributions are Kocherlakota and Kocherlakota [6] and Johnson et al. [7]. Lakshminarayana et al. [8] have proposed a bivariate Poisson distribution as a product of Poisson marginals with taking an account of positive, zero or negative correlation between two variables depends on multiplicative factor parameter. Supanekar and Shirke [9] have proposed a new bivariate generalized power series distribution. Vanitha et al. [10] are proposed a bivariate beta mixture model for segmenting the medical images by considering the Bivariate features.

In this paper we construct bivariate discrete distribution from bivariate continuous distribution by taking integer part of variables. Rest of the paper is organized as follows; the next
Section II contributes to construction of bivariate discrete distribution by taking integer part of variables of bivariate exponential distribution due to Freund [11]. Section III describes the marginal probability mass functions obtained from bivariate discrete distribution introduced in Section II. In the last Section IV concludes research work.

\section{BIVARIATE DISCRETE DISTRIBUTION}

Suppose random vector (U, V) has bivariate exponential distribution given by Freund [11] with probability density function

$$
f(u, v)= \begin{cases}\theta_{1} \theta_{2}^{\prime} e^{-\theta_{2}^{\prime} v-\left(\theta_{1}+\theta_{2}-\theta_{2}^{\prime}\right) u}, & 0<u<v \\ \theta_{2} \theta_{1}^{\prime} e^{-\theta_{1}^{\prime} u-\left(\theta_{1}+\theta_{2}-\theta_{1}^{\prime}\right) v}, & 0<v<u\end{cases}
$$

where $\theta_{1}, \theta_{1}^{\prime}, \theta_{2}, \theta_{2}^{\prime}>0$.

In the following we discuss bivariate discrete distribution derived from Freund bivariate exponential distribution given in (1)

\section{Theorem 1.}

Let $\mathrm{X}$ be the integer part of $\mathrm{U}$ and $\mathrm{Y}$ be the integer part of $\mathrm{V}$, then the joint probability mass function of $(X, Y)$ is given by 


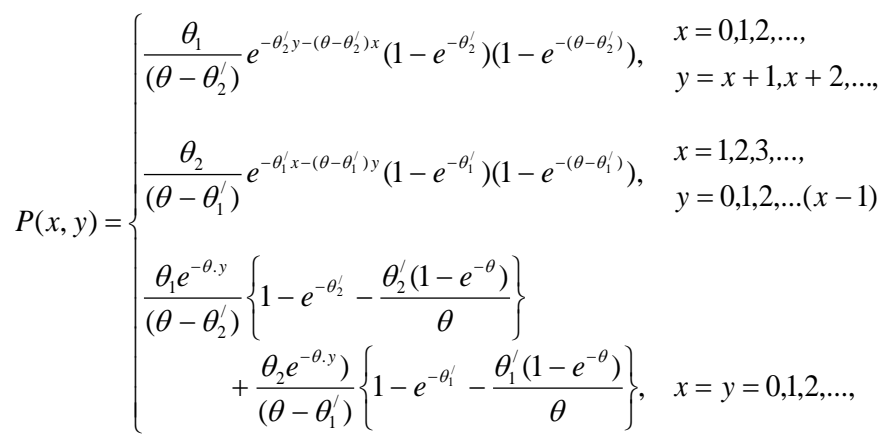

where $\theta_{1}, \theta_{1}^{\prime}, \theta_{2}, \theta_{2}^{\prime}>0$ and $\theta=\theta_{1}+\theta_{2}$.

Proof : We divide the sample space into three disjoint regions as follows

Case I: $R_{1}=\{(x ; y): x<y\}$.

Equivalently, $R_{1}=\{(u ; v): 0<u<v\}$

Case II: $R_{2}=\{(x ; y): \mathrm{y}<x\}$.

Equivalently, $R_{2}=\{(u ; v): 0<v<u\}$

Case III: $R_{3}=\{(x ; y): x=y\}$.

Equivalently, $R_{3}=\{(u ; v): 0<u<v$ or $0<v<u\}$

In the following joint probability mass function of $(\mathrm{X}, \mathrm{Y})$ is obtained for each region.

Case I : Let $(x, y) \in R_{1}$. Then

$$
\begin{aligned}
P(X=x, Y=y) & =P(x \leq U<x+1, y \leq V<y+1) \\
= & \int_{x}^{x+1} \int_{y}^{y+1} f(u, v) d u . d v \\
= & \int_{x}^{x+1} \int_{y}^{y+1} \theta_{1} \theta_{2}^{\prime} e^{-\theta_{2}^{\prime} v-\left(\theta_{1}+\theta_{2}-\theta_{2}^{\prime}\right) u} d u . d v \\
= & \frac{\theta_{1}}{\left(\theta-\theta_{2}^{\prime}\right)} e^{-\theta_{2}^{\prime} y-\left(\theta-\theta_{2}^{\prime}\right) x}\left(1-e^{-\theta_{2}^{\prime}}\right)\left(1-e^{-\left(\theta-\theta_{2}^{\prime}\right)}\right) .
\end{aligned}
$$

Case II : Let $(x, y) \in R_{2}$. Then

$$
\begin{aligned}
P(X=x, Y=y)= & P(x \leq U<x+1, y \leq V<y+1) \\
& =\int_{x}^{x+1} \int_{y}^{y+1} f(u, v) d u . d v \\
& =\int_{x}^{x+1} \int_{y}^{y+1} \theta_{2} \theta_{1}^{\prime} e^{-\theta_{1}^{\prime} u-\left(\theta_{1}+\theta_{2}-\theta_{1}^{\prime}\right) v} d u . d v \\
& =\frac{\theta_{2}}{\left(\theta-\theta_{1}^{\prime}\right)} e^{-\theta_{1}^{\prime} x-\left(\theta-\theta_{1}^{\prime}\right) y}\left(1-e^{-\theta_{1}^{\prime}}\right)\left(1-e^{-\left(\theta-\theta_{1}^{\prime}\right)}\right) .
\end{aligned}
$$

Case III : Let $(x, y) \in R_{3}$. Then

$$
\begin{aligned}
& P(X=x, Y=y)=P(x \leq U<x+1, y \leq V<y+1) \\
&= \underbrace{\int_{y}^{y+1} \int_{y}^{v} f(u, v) d u \cdot d v}_{u<v}+\underbrace{\int_{y}^{y+1} \int_{y}^{u} f(u, v) d u . d v}_{v<u} \\
&= \int_{y}^{y+1} \int_{y}^{v} \theta_{1} \theta_{2}^{\prime} e^{-\theta_{2}^{\prime} v-\left(\theta_{1}+\theta_{2}-\theta_{2}^{\prime}\right) u} d u \cdot d v \\
& \quad+\int_{y}^{y+1} \int_{y}^{u} \theta_{2} \theta_{1}^{\prime} e^{-\theta_{1}^{\prime} u-\left(\theta_{1}+\theta_{2}-\theta_{1}^{\prime}\right) v} d u \cdot d v \frac{\theta_{1} e^{-\theta y}}{\left(\theta-\theta_{2}^{\prime}\right)}\left\{1-e^{-\theta_{2}^{\prime}}-\frac{\theta_{2}^{\prime}\left(1-e^{-\theta}\right)}{\theta}\right\} \\
&+\frac{\theta_{2} e^{-\theta y}}{\left(\theta-\theta_{1}^{\prime}\right)}\left\{1-e^{-\theta_{1}^{\prime}}-\frac{\theta_{1}^{\prime}\left(1-e^{-\theta}\right)}{\theta}\right\} .
\end{aligned}
$$

From equations in (3) to (5), we get the joint probability mass function given in (2).

\section{MARGINAL PROBABILITY MASS FUNCTIONS}

In this Section, we discuss marginal distributions of bivariate discrete distribution given in (2)

\section{Lemma 1.}

1) Marginal probability mass function of $X$ is given by

$$
\begin{aligned}
P(X=x)= & \frac{\theta_{2}}{\left(\theta-\theta_{1}^{\prime}\right)}\left(1-e^{-\theta_{1}^{\prime}}\right) e^{-\theta_{1}^{\prime} x} \\
& +\left(\frac{\theta_{1}-\theta_{1}^{\prime}}{\theta-\theta_{1}^{\prime}}\right)\left(1-e^{-\theta}\right) e^{-\theta x}, x=0,1,2, \ldots
\end{aligned}
$$

2) Marginal probability mass function of $Y$ is given by

$$
\begin{aligned}
P(Y=y)= & \frac{\theta_{1}}{\left(\theta-\theta_{2}^{\prime}\right)}\left(1-e^{-\theta_{2}^{\prime}}\right) e^{-\theta_{2}^{\prime} y} \\
& +\left(\frac{\theta_{2}-\theta_{2}^{\prime}}{\theta-\theta_{2}^{\prime}}\right)\left(1-e^{-\theta}\right) e^{-\theta y}, y=0,1,2, \ldots
\end{aligned}
$$

Proof: The marginal probability mass function of $\mathrm{X}$ is given by

$$
\begin{aligned}
P(X=x) & =\underbrace{\sum_{y=0}^{x-1} P(X=x, Y=y)}_{y<x}+\underbrace{P(X=x, Y=y)}_{y=x}+\underbrace{\sum_{y=x+1}^{\infty} P(X=x, Y=y)}_{y>x} \\
& =S_{1}+S_{2}+S_{3}
\end{aligned}
$$


Now,

$$
\begin{aligned}
S_{1} & =\sum_{y=0}^{x-1} P(X=x, Y=y) \\
& =\sum_{y=0}^{x-1} \frac{\theta_{2}}{\left(\theta-\theta_{1}^{\prime}\right)} e^{-\theta_{1}^{\prime} x-\left(\theta-\theta_{1}^{\prime}\right) y}\left(1-e^{-\theta_{1}^{\prime}}\right)\left(1-e^{-\left(\theta-\theta_{1}^{\prime}\right)}\right) \\
& =\frac{\theta_{2}}{\left(\theta-\theta_{1}^{\prime}\right)}\left(1-e^{-\theta_{1}^{\prime}}\right)\left(1-e^{-\left(\theta-\theta_{1}^{\prime}\right)}\right) e^{-\theta_{1}^{\prime} x} \sum_{y=0}^{x-1} e^{-\left(\theta-\theta_{1}^{\prime}\right) y} \\
& =\frac{\theta_{2}}{\left(\theta-\theta_{1}^{\prime}\right)}\left(1-e^{-\theta_{1}^{\prime}}\right)\left(e^{-\theta_{1}^{\prime} x}-e^{-\theta x}\right),
\end{aligned}
$$

$$
\begin{aligned}
& S_{2}=P(X=x, Y=x) \\
& =\frac{\theta_{1} e^{-\theta \cdot x}}{\left(\theta-\theta_{2}^{\prime}\right)}\left\{1-e^{-\theta_{2}^{\prime}}-\frac{\theta_{2}^{\prime}\left(1-e^{-\theta}\right)}{\theta}\right\} \\
& \quad+\frac{\theta_{2} e^{-\theta \cdot x}}{\left(\theta-\theta_{1}^{\prime}\right)}\left\{1-e^{-\theta_{1}^{\prime}}-\frac{\theta_{1}^{\prime}\left(1-e^{-\theta}\right)}{\theta}\right\}
\end{aligned}
$$

and

$$
\begin{aligned}
S_{3} & =\sum_{y=x+1}^{\infty} P(X=x, Y=y) \\
& =\sum_{y=x+1}^{\infty} \frac{\theta_{1}}{\left(\theta-\theta_{2}^{\prime}\right)} e^{-\theta_{2}^{\prime} y-\left(\theta-\theta_{2}^{\prime}\right) x}\left(1-e^{-\theta_{2}^{\prime}}\right)\left(1-e^{-\left(\theta-\theta_{2}^{\prime}\right)}\right) \\
& =\frac{\theta_{1}}{\left(\theta-\theta_{2}^{\prime}\right)}\left(1-e^{-\theta_{2}^{\prime}}\right)\left(1-e^{-\left(\theta-\theta_{2}^{\prime}\right)}\right) e^{-\left(\theta-\theta_{2}^{\prime}\right) x} \sum_{y=x+1}^{\infty} e^{-\theta_{2}^{\prime} y} \\
& =\frac{\theta_{1} e^{-\theta x}}{\left(\theta-\theta_{2}^{\prime}\right)}\left(e^{-\theta_{2}^{\prime}}-e^{-\theta}\right) .
\end{aligned}
$$

Putting $S_{1}, S_{2}$ and $S_{3}$ in (8) we get,

$$
\begin{aligned}
P(X=x)= & \frac{\theta_{2}}{\left(\theta-\theta_{1}^{\prime}\right)}\left(1-e^{-\theta_{1}^{\prime}}\right)\left(e^{-\theta_{1}^{\prime} x}-e^{-\theta x}\right) \\
& +\frac{\theta_{1} e^{-\theta \cdot x}}{\left(\theta-\theta_{2}^{\prime}\right)}\left\{1-e^{-\theta_{2}^{\prime}}-\frac{\theta_{2}^{\prime}\left(1-e^{-\theta}\right)}{\theta}\right\} \\
& +\frac{\theta_{2} e^{-\theta \cdot x}}{\left(\theta-\theta_{1}^{\prime}\right)}\left\{1-e^{-\theta_{1}^{\prime}}-\frac{\theta_{1}^{\prime}\left(1-e^{-\theta}\right)}{\theta}\right\} \\
& +\frac{\theta_{1} e^{-\theta x}}{\left(\theta-\theta_{2}^{\prime}\right)}\left(e^{-\theta_{2}^{\prime}}-e^{-\theta}\right)
\end{aligned}
$$

$$
=\frac{\theta_{2}}{\left(\theta-\theta_{1}^{\prime}\right)}\left(1-e^{-\theta_{1}^{\prime}}\right) e^{-\theta_{1}^{\prime} x}+\left(\frac{\theta_{1}-\theta_{1}^{\prime}}{\theta-\theta_{1}^{\prime}}\right)\left(1-e^{-\theta}\right) e^{-\theta x} .
$$

Hence marginal probability mass function of $\mathrm{X}$ is given by

$$
\begin{aligned}
P(X=x)= & \frac{\theta_{2}}{\left(\theta-\theta_{1}^{\prime}\right)}\left(1-e^{-\theta_{1}^{\prime}}\right) e^{-\theta_{1}^{\prime} x} \\
& +\left(\frac{\theta_{1}-\theta_{1}^{\prime}}{\theta-\theta_{1}^{\prime}}\right)\left(1-e^{-\theta}\right) e^{-\theta x}, x=0,1,2, \ldots
\end{aligned}
$$

Similarly marginal probability mass function of $\mathrm{Y}$ is given by

$$
\begin{aligned}
P(Y=y)= & \frac{\theta_{1}}{\left(\theta-\theta_{2}^{\prime}\right)}\left(1-e^{-\theta_{2}^{\prime}}\right) e^{-\theta_{2}^{\prime} y} \\
& \quad+\left(\frac{\theta_{2}-\theta_{2}^{\prime}}{\theta-\theta_{2}^{\prime}}\right)\left(1-e^{-\theta}\right) e^{-\theta y}, y=0,1,2, \ldots
\end{aligned}
$$

Hence proof.

\section{Remark 1:}

By letting,

$$
\begin{aligned}
& \frac{\theta_{2}}{\left(\theta-\theta_{1}^{\prime}\right)}=w_{1},\left(\frac{\theta_{1}-\theta_{1}^{\prime}}{\theta-\theta_{1}^{\prime}}\right)=w_{2}, \quad \text { where } w_{1}+w_{2}=1 \text {, } \\
& \frac{\theta_{1}}{\left(\theta-\theta_{2}^{\prime}\right)}=w_{3},\left(\frac{\theta_{2}-\theta_{2}^{\prime}}{\theta-\theta_{2}^{\prime}}\right)=w_{4}, \quad \text { where } w_{3}+w_{4}=1 \text {, } \\
& \left(1-e^{-\theta_{1}^{\prime}}\right)=p_{1}, \quad e^{-\theta_{1}^{\prime}}=q_{1}, \quad \text { where } p_{1}+q_{1}=1 \text {, } \\
& \left(1-e^{-\theta_{2}^{\prime}}\right)=p_{2}, \quad e^{-\theta_{2}^{\prime}}=q_{2}, \quad \text { where } p_{2}+q_{2}=1 \text {, } \\
& \left(1-e^{-\theta}\right)=p_{3}, \quad e^{-\theta}=q_{3}, \quad \text { where } p_{3}+q_{3}=1 \text {, }
\end{aligned}
$$

The marginal probability mass function of $\mathrm{X}$ and $\mathrm{Y}$ can be rewritten as

The marginal probability mass function of $\mathrm{X}$ is given by

$$
P(X=x)=w_{1} p_{1} q_{1}^{x}+w_{2} p_{3} q_{3}{ }^{x}, \quad x=0,1,2, \ldots
$$

And marginal probability mass function of $\mathrm{Y}$ is given by

$$
P(Y=y)=w_{3} p_{2} q_{2}{ }^{y}+w_{4} p_{3} q_{3}{ }^{y}, \quad y=0,1,2, \ldots
$$

Here we get weighted geometric distributions. 


\section{CONCLUSION}

In this paper a bivariate discrete distribution is introduced. This distribution is constructed by transforming a bivariate continuous random vector to a bivariate discrete random vector. Continuous random vector has been transformed to a discrete random vector by considering integer part of the continuous random vector. Freund bivariate exponential distribution is considered and corresponding bivariate discrete distribution is obtained. This bivariate discrete distribution is new in the literature. Marginal distributions of newly defined bivariate discrete distribution are also derived. There is wide scope to study this newly defined bivariate discrete distribution.

\section{REFERENCES}

[1] E. Gomez-Deniz, F. J. Vazquez-Polo and V. Garcia-Garcia, " $A$ discrete version of the half- normal distribution and its generalization with applications", Stat Papers, Vol.55, pp.497511,2014

[2] P. Holgate, "Estimation for the bivariate Poisson distribution", Biometrika, Vol.51, pp.241-245, 1964.

[3] H. Teicher, "On the multivariate Poisson distribution", Skandinavisk Aktuarietidskrift, Vol.37, pp.1-9, 1954

[4] M. A. Hamdan, "Estimation in the truncated bivariate Poisson distribution", Technometrics, Vol.14, pp.33-45, 1972.

[5] R. C. Dahiya, "Estimation in a truncated bivariate Poisson distribution", Communications in Statistics Theory and Methods, Vol.6, pp.113-120, 1977.

[6] S. Kocherlakota, K. Kocherlakota, "Bivariate Discrete Distributions", Marcel Dekker, New York, 1992
[7] N. L. Johnson, S. Kotz, and N. Balakrishnan, "Discrete multivariate distributions", John Wiley and Sons, New York, 1997.

[8] J. Lakshminarayana, S. N. N. Pandit, K. Rao, Srinivasa, "On Bivariate Poisson distribution", Commun. Statistics-Theory Methods, Vol.28(2), pp.267-276, 1999

[9] S. R. Supanekar and D. T. Shirke, "A new bivariate generalized power series distributiion”, Int. J. Agricult. Stat. Sci., Vol. 10(2), pp.343-349, 2014

[10] K.Vanitha, M. Suresh Kumar, Sk. Althaf Rahaman, "An Effective Segmentation Approach of Medical Images Based on Beta Mixture Model", International Journal of Computer Sciences and Engineering, Vol.6 (3), pp.225-229, 2018.

[11] J. E. Freund, "A bivariate extension of the exponential distribution", J. Amer. Statist. Assoc., Vol.56, pp.971-977, 1961.

\section{AUTHOR PROFILE}

Mr. S. R. Supanekar completed M.Sc. and Ph.D. in Statistics from Shivaji University Kolhapur in 1993 \& 2016. He is currently working as Assistant Professor in Department of Statistics, Balasaheb Desai College, Patan, Dist. Satara, India (MS). He has published 3 research papers in reputed international journals. His main research work focuses on discrete distributions. He has 21 years of teaching experience and 6 years of research experience. 